\title{
Clinical outcome observation of preoperative concurrent chemoradiotherapy/radiotherapy alone in 174 Chinese patients with local advanced cervical carcinoma
}

\author{
This article was published in the following Dove Press journal: \\ OncoTargets and Therapy \\ 5 February 2013 \\ Number of times this article has been viewed
}

\author{
Li-Chun Weil,* \\ Ning Wangl,* \\ Mei Shi' \\ Jun-Yue Liu' \\ Jian-Ping $\mathrm{Li}^{\prime}$ \\ Ying Zhang' \\ Yan-Hong Huang ${ }^{2}$ \\ Xia $\mathrm{Li}^{3}$ \\ Yan Chen ${ }^{4}$ \\ 'Department of Radiation Oncology, \\ ${ }^{2}$ Department of Gynecology and \\ Obstetrics, ${ }^{3}$ Department of Pathology, \\ ${ }^{4}$ Department of Oncology, Xijing \\ Hospital, Xi'an, People's Republic \\ of China \\ *Shared first co-authorship
}

\begin{abstract}
Objective: To study outcomes of concurrent chemoradiotherapy (CCRT) or radiotherapy (RT) alone followed by radical surgery in patients with local advanced cervical cancer.

Methods: A retrospective approach was carried out in 174 Chinese patients with International Federation of Obstetricians and Gynaecologists stage IB2-IIIB cervical carcinoma. A total of 121 patients were treated with CCRT, while the remaining 53 patients received RT alone, and the regimen of chemotherapy was weekly cisplatin $\left(40 \mathrm{mg} / \mathrm{m}^{2}\right)$. Pathological response, overall survival (OS), progression-free survival (PFS), and complications were analyzed.
\end{abstract}

Results: The median age was 45 years and the mean primary tumor diameter was $4.8 \pm 1.0 \mathrm{~cm}$. Pathological complete response (CR) was achieved in 53 patients $(30.5 \%)$. The CR rate was relatively higher in the CCRT group $(31.4 \%$ vs $28.3 \%, P=0.724)$, particularly when tumor diameter was less than $5 \mathrm{~cm}$ (38.2\% vs 30.8\%, $P=0.623)$. With median follow-up of 24 months, patients with CR had improved 3-year OS $(100 \%$ vs $83.6 \%, P=0.018)$ and 3-year PFS $(93.1 \%$ vs $83.2 \%, P=0.035)$ compared to patients with residual disease. CCRT was associated with significantly improved 3 -year PFS $(92.0 \%$ vs $76.5 \%, P=0.032)$ compared to RT alone in patients with tumor diameter less than $5 \mathrm{~cm}$. Thirty-seven patients $(21.3 \%)$ experienced more than grade 2 toxicity, and one patient $(0.6 \%)$ developed grade 3 uronephrosis. Data thus indicated that pathologic response, tumor size, and lymph-node involvement were highly correlated with clinical outcomes of the local advanced cervical disease.

Conclusion: Preoperative CCRT achieved outcomes superior to RT alone, depending on the pathologic response, tumor size and lymph-node involvement as major prognostic factors.

Keywords: local advanced cervical carcinoma, concurrent chemoradiotherapy, radical hysterectomy, pelvic lymphadenectomy, prognostic factors

\section{Introduction}

Cervical carcinoma represents the second-leading cancer affecting women's health worldwide, and is more serious in China, especially in the northwest regions. Although concomitant chemoradiotherapy (CCRT) has been recommended as standard treatment for local advanced cervical carcinoma (LACC) since 1999, 5-year overall survival (OS) of LACC patients is not satisfactory presently, and it is still crucial to explore a more effective therapeutic strategy for further OS improvement of LACC. ${ }^{1-6}$ The treatment strategy of cervical carcinoma has been changed significantly in the past two decades. Cisplatin-based CCRT has become the standard of care for LACC patients in most developed counties in the world. ${ }^{7}$ CCRT can reduce tumor recurrence and
Correspondence: Mei Shi Department of Radiation Oncology, Xijing Hospital, Fourth Military Medical University, 169 Changle West Road, Xi'an 710032, People's Republic of China Tel +862984775432

Email mshi82@fmmu.edu.cn 
metastasis by $30 \%-50 \%$ and improve OS by $9 \%-18 \%$ as well. ${ }^{8}$ Nevertheless, it is still not clear whether CCRT can provide a significant advantage for LACCs, eg, stages IIIIVA, in comparison with radiotherapy (RT) alone or in combination following radical surgery. ${ }^{3,9}$

Several previous observations focusing on neoadjuvant CCRT followed by surgery were reported, with findings that the extent of postoperative pathological response impacted on OS and progression-free survival (PFS) of patients with cervical cancer. ${ }^{2,10,11}$ A meta-analysis study also demonstrated that survival benefit of CCRT might be restricted to lowerstage patients with International Federation of Obstetricians and Gynaecologists (FIGO) stage IB-IIA, IIB having an increase in OS of $10 \%$ and $7 \%$, respectively, by CCRT. ${ }^{12}$ However, these previous studies did not define effects of primary tumor size, treatment modality, and preoperative radiation dose on pathological response and survival of patients. Therefore, the aim of this study is to observe if any different outcomes may occur between CCRT and RT alone followed by radical surgery in patients with FIGO stage IB2IIIB cervical cancer by analysis of postoperative pathologic response, tumor diameter, and lymph-node involvement as major prognostic factors.

\section{Materials and methods}

\section{Patient population}

From April 2006 to June 2011, 174 patients with cervical carcinoma (FIGO stage IB2-IIIB, according to the pelvic examination) were treated in the Department of Radiation Oncology, Xijing Hospital, Fourth Military Medical University, China. Pretreatment evaluations were included as follows: patient's disease history, blood counts, liver and renal function tests, gynecological examination, tumor biopsy, chest X-ray, transvaginal ultrasound (TVS), transabdominal ultrasonography, and/or pelvic magnetic resonance imaging (MRI). Patient characteristics are shown in detail in Table 1. Tumor sizes were basically determined by TVS examination. All patients had Karnofsky performance status $\geq 70$ and had no history of other malignancy or cancer therapy.

\section{Neoadjuvant chemoradiotherapy or radiotherapy alone}

Preoperative pelvic RT was delivered using threedimensional conformal radiation techniques and 6 or $15 \mathrm{MV}$ photons using a linear accelerator (Clinac 23EX or $600 \mathrm{C} / \mathrm{D}$; Varian Medical Systems, Palo Alto, CA, USA). Patients were immobilized with a custom vacuum mattress in the supine position and underwent a computed tomography (CT) simulation scan (PQS + AcQSim; Philips, Amsterdam, Netherlands) with intravenous contrast, using $5 \mathrm{~mm}$ slice thickness. Simulation images extended from L1 to $5 \mathrm{~cm}$ below the ischial tuberosities.

The clinical target volume (CTV) included the gross tumor, cervix, uterus, parametria, the upper part of the vagina to $3 \mathrm{~cm}$ below the tumor invasion (according to T2-weighted MRI image), and regional lymph nodes (common, external,

Table I Characteristics of I74 patients with IB2-IIIB cervical carcinoma

\begin{tabular}{|c|c|c|c|}
\hline Characteristics & Number of patients (\%) & Number of CCRT (\%) & Number of RT alone (\%) \\
\hline Median age (range, years) & $45(25-66)$ & $45(25-65)$ & $45(34-66)$ \\
\hline \multicolumn{4}{|l|}{ FIGO stages } \\
\hline IB2 & $4(2.3 \%)$ & $3(2.5 \%)$ & $\mathrm{I}(\mathrm{I} .9 \%)$ \\
\hline IIB & $158(90.8 \%)$ & 110 (90.9\%) & $48(90.6 \%)$ \\
\hline IIIB & $12(6.9 \%)$ & $8(6.6 \%)$ & $4(7.5 \%)$ \\
\hline Tumor diameters & $(4 . I-8,5,4.8)$ & $(4-8,5,4.8)$ & $(4-6.3,4.9,4.7)$ \\
\hline \multicolumn{4}{|l|}{ (range, median, mean) } \\
\hline$\leq 4.9 \mathrm{~cm}$ & 81 (46.6\%) & 55 (45.5\%) & $26(49.1)$ \\
\hline $5.0-5.9 \mathrm{~cm}$ & 75 (43.1\%) & $56(46.3 \%)$ & $19(35.8 \%)$ \\
\hline$\geq 6 \mathrm{~cm}$ & $18(10.3 \%)$ & $10(8.3 \%)$ & $8(15.1 \%)$ \\
\hline \multicolumn{4}{|l|}{ Preoperative pathological types } \\
\hline Squamous cell carcinoma & $163(93.7 \%)$ & 114 (94.2\%) & 49 (92.5\%) \\
\hline Nonsquamous cell carcinoma & $11(6.3 \%)$ & $7(5.8 \%)$ & $4(7.5 \%)$ \\
\hline \multicolumn{4}{|l|}{ Preoperative EBRT doses } \\
\hline $40-45$ Gy/20-25 f & $66(37.9 \%)$ & 30 (24.8\%) & $36(67.9 \%)$ \\
\hline $46-50 \mathrm{~Gy} / 23-25 \mathrm{f}$ & $108(62.1 \%)$ & 91 (75.2\%) & $17(32.1 \%)$ \\
\hline \multicolumn{4}{|l|}{ RT mode } \\
\hline With brachytherapy & $16(9.2 \%)$ & $11(9.1 \%)$ & $5(9.4 \%)$ \\
\hline Without brachytherapy & I 58 (90.8\%) & 110 (90.9\%) & $48(90.6 \%)$ \\
\hline
\end{tabular}

Abbreviations: CCRT, concurrent chemoradiotherapy; EBRT, external beam radiotherapy; RT, radiotherapy. 
internal iliac lymph nodes, obturator and presacral lymph nodes). The lymph nodes were delineated according to guidelines for delineation in pelvic intensity-modulated radiotherapy (IMRT). ${ }^{13}$ The planning target volume (PTV) was defined by a uniform three-dimensional expansion around the CTV, using $7 \mathrm{~mm}$ margins around the lymph nodes; $10 \mathrm{~mm}$ around the vagina and, parametria; and $15 \mathrm{~mm}$ around the cervix and gross disease. The treatment planning was designed and computed using the Plato system version 2.7.5 (Varian). Pelvic irradiation dose was 40-50 Gy in 20-25 fractions.

The CT-based image-guided brachytherapy was applied to treat 16 patients with large and poorly responding tumors at the conclusion of pelvic external beam radiotherapy (EBRT). High-dose-rate brachytherapy was delivered with 5-22 Gy in 1-3 fractions to $90 \%$ of the high-risk CTV ( $\mathrm{D}_{90}$ for HR-CTV), using interstitial implantation or an intracavity applicator (microSelectron-HDR Ir-192 set or the Fletcher applicator set; Nucletron, Veenendaal, Netherlands). The CCRT was administered to 121 patients by an intravenous infusion of weekly cisplatin $\left(40 \mathrm{mg} / \mathrm{m}^{2}\right)$ during pelvic EBRT. Patients received either three $(n=30,25 \%)$, four $(n=46$, $38 \%)$, or five ( $n=45,37 \%)$ cycles of cisplatin. Chemotherapy was held under the following conditions: white blood cell count $<2.0 \times 10^{9} / \mathrm{L}$, absolute neutrophil count $<1.0 \times 10^{9} / \mathrm{L}$, platelet count $<50 \times 10^{9} / \mathrm{L}$ or grade $3-4$ radiation enteritis or cystitis.

\section{Surgery and histopathological examination}

All patients underwent radical abdominal hysterectomy and pelvic lymphadenectomy, with the median number of lymph nodes removed being 18 (range 6-35). The interval between preoperative RT and radical surgery was $2-3$ weeks. The resected primary and lymph-node specimens were prepared on hematoxylin and eosin stain slides. Pathological response to neoadjuvant therapy was evaluated based on the histopathological examination of resected specimens (ie, uterus, vaginal cuff, parametria, pelvic lymph nodes). Pathological response of primary tumor was classed as pathological complete response (CR), partial response (PR), or residual carcinoma (RC). Pathological response of resected lymph nodes was described in terms of lymph-node involvement (involved or not involved).

\section{Follow-up observations}

Toxicity assessment was performed according to the Radiation Therapy Oncology Group/European Organization for
Research and Treatment for Cancer late-radiation morbidityscoring scheme. ${ }^{14}$ Surgical complications were classified by the Chassagne grading system. ${ }^{15,16}$ After completion of treatment, patients were followed at 3-month intervals for the first 12 months, and at 6-month intervals thereafter. Patients were followed up regularly with gynecological examination, laboratory studies (blood counts, liver and renal function tests), TVS, transabdominal ultrasonography, superficial lymph-node examination, and radiographic studies, such as chest CT and/or pelvic MRI.

\section{Data collection and statistical analysis}

The pelvic tumor control, OS, and PFS were calculated from the date of surgery to the last date of follow-up. Death in the absence of progression was censored in the calculation of PFS. The Kaplan-Meier method and log-rank test were used to estimate outcomes and effects on OS and PFS, respectively. Multivariate analysis of prognostic factors was performed with Cox proportional hazards regression. The chi-squared test was used to compare proportions between different groups. SPSS (IBM, Armonk, NY, USA) software was used for all statistical analyses, and $P$-values less than 0.05 were considered statistically significant.

\section{Results \\ Patient characteristics and postoperative pathologic response}

Median age of 174 patients was 45 years (range 25-66). The mean tumor diameter was $4.8 \pm 1.0 \mathrm{~cm}$ (range $4.1-8 \mathrm{~cm}$ ), as measured by TVS. Pathologic subtypes were predominantly squamous cell carcinoma $(n=163,93.7 \%)$, with a minority having adenocarcinoma $(n=8,4.6 \%)$ or adenosquamous carcinoma $(n=3,1.7 \%)$. The characteristics and postoperative pathological response of 174 Chinese patients with LACC are summarized in Table 1.

\section{Survival outcomes of CCRT in comparison with RT alone}

Median follow-up from the date of surgery was 24 months (range 4-68 months). The 3-year PFS and OS were 90.6\% and $93.1 \%$, respectively (Figure 1). The 3-year local pelvic control was $97.1 \%(n=169)$. Death was reported in eleven patients $(11 / 174,6.3 \%)$, from distant metastasis $(7 / 174$, $4.0 \%)$, pelvic recurrence $(2 / 174,1.1 \%)$, severe complication of renal failure $(1 / 174,0.6 \%)$, and second primary cancer $(1 / 174,0.6 \%)$. There was no statistical significance between CCRT and RT alone in metastasis rate (3/121, 2.5\% vs $4 / 53,7.5 \% ; P=0.202)$ and local recurrence rate $(2 / 121$, 


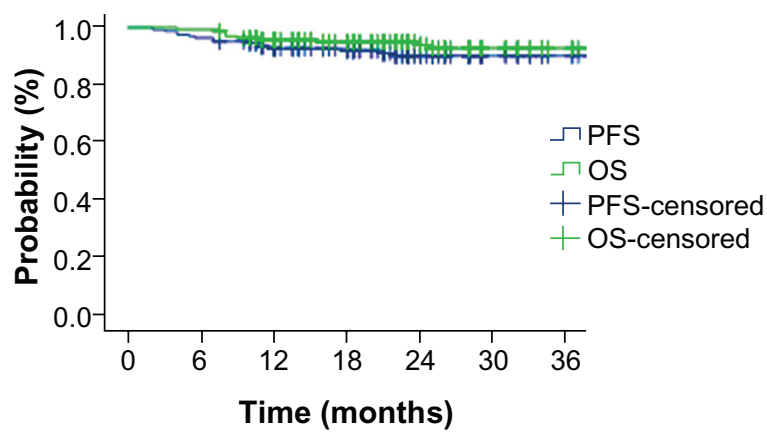

$\begin{array}{cccc}\text { No at risk } & \mathbf{1 2} \mathbf{~} & \mathbf{2 4} \mathbf{~} & \mathbf{3 6} \mathbf{~} \\ \text { OS } & 167 & 165 & 164 \\ \text { PFS } & 162 & 159 & 159\end{array}$

Figure I Kaplan-Meier survival curves of 174 patients treated with concurrent chemoradiotherapy or radiotherapy alone followed by a radical hysterectomy. Note: The 3-year follow-up indicates that PFS is $90.6 \%$ and OS is $93.1 \%$. Abbreviations: OS, overall survival; PFS, progression-free survival.

$1.7 \%$ vs $0 / 53,0 \% ; P=1.000)$, but the tendency showed CCRT could possibly decrease the metastasis rate. In order to exclude the confounding factor, subgroup analysis classified by tumor size and postoperative pathologic response were performed.

On multivariate analysis, the following prognostic factors were highly correlated with survival: primary tumor diameter, age, postoperative pathologic response, and pelvic lymphnode involvement status (Table 2 and Figure 2). Positive lymph-node involvements were confirmed in 15 patients (twelve patients in the CCRT and three patients in the RT alone group). There was no statistically significant difference in lymph-node involvement status between CCRT and RT alone $(12 / 121,9.9 \%$ vs $3 / 53,5.7 \% ; P=0.058)$.

OS was not significantly associated with pathologic subtype (squamous cell carcinoma vs nonsquamous cell carcinoma, 3-year PFS, 93.1\% vs 91.7\%, $P=0.527$; 3-year OS, $93.3 \%$ vs $91.7 \%, P=0.588$ ) or FIGO stage (IB2-IIB vs IIIB, 3-year PFS, $89.9 \%$ vs $100 \%, P=0.461$; 3-year OS, $92.5 \%$ vs $100 \%, P=0.517$ ). The treatment modality (CCRT vs RT alone, 3-year PFS, $92.0 \%$ vs $86.7 \%, P=0.069$; 3-year OS, $95.0 \%$ vs $88.1 \%, P=0.124)$ and preoperative EBRT dose (40-45 Gy vs 46-50 Gy, 3-year PFS, 93.2\% vs 89.0\%, $P=0.094$; 3-year OS, $94.6 \%$ vs $91.9 \%, P=0.177$ ) were not significantly associated with OS and PFS of patients with LACC (Table 2).

To observe any complications with the median follow-up period of 24 months, 37 of the patients experienced late grade $\geq 2$ complications. Seven patients $(7 / 174,4.0 \%)$ experienced grade 3 complications, without significant differences between the CCRT and RT-alone groups $(21.5 \%$ vs $20.8 \%, P=0.782$ ). Among these, one patient developed renal failure due to severe uronephrosis, six patients experienced two complications, and one patient experienced three complications (Table 3).

\section{Pathologic response as one key prognostic factor in CCRT and RT alone}

The postoperative pathologic response included $\mathrm{CR}, \mathrm{PR}$, and RC. CR was defined as a complete disappearance of all macroscopic and microscopic diseases and mainly showing inflammatory cell infiltration, PR defined as presence of persistent atypical cells or cervical intraepithelial neoplasia, and $\mathrm{RC}$ defined as macroscopic and/or microscopic residual disease (Figure 2). The CR rate was 30.5\% (53/174), PR rate was $31.6 \%(55 / 174)$, and $\mathrm{RC}$ rate was $37.9 \%(66 / 174)$. Among patients with RC, 18 had greater than one-third cervical stromal invasion, four developed capillary-like space involvement, and one had ovarian invasion. Survival status was benefited in the CCRT group in comparison with that of the RC-alone group $(P=0.035,94.9 \%$ vs $84.0 \%$ for 3 -year PFS; $P=0.018,100 \%$ vs $87.3 \%$ for 3 -year OS) (Figure 3 ).

Table 2 Correlations of major clinical/pathological factors and overall survival

\begin{tabular}{|c|c|c|c|c|c|c|c|c|}
\hline \multirow[t]{2}{*}{ Factors } & \multirow{2}{*}{$\begin{array}{l}P \text {-value } \\
\text { (3-year OS ) }\end{array}$} & \multirow[t]{2}{*}{$\operatorname{Exp}(B) H R$} & \multicolumn{2}{|l|}{$95 \% \mathrm{Cl}$} & \multirow{2}{*}{$\begin{array}{l}P \text {-value } \\
\text { (3-year PFS) }\end{array}$} & \multirow[t]{2}{*}{$\operatorname{Exp}(B) H R$} & \multicolumn{2}{|l|}{$95 \% \mathrm{Cl}$} \\
\hline & & & Lower & Upper & & & Lower & Upper \\
\hline \multicolumn{9}{|l|}{ Tumor diameters } \\
\hline$(\geq 5 \mathrm{~cm}$ vs $<5 \mathrm{~cm})$ & 0.059 & 0.263 & 0.066 & 1.054 & 0.024 & 0.251 & 0.076 & 0.835 \\
\hline \multicolumn{9}{|l|}{ Age of patients } \\
\hline ( $\leq 35$ years vs $>35$ years $)$ & 0.089 & 0.308 & 0.080 & $\mathrm{I} .195$ & 0.049 & 0.312 & 0.098 & 0.995 \\
\hline Postoperative lymph-node & 0.087 & 0.248 & 0.050 & 1.223 & 0.001 & 0.135 & 0.040 & 0.456 \\
\hline \multicolumn{9}{|c|}{ involvement (positive vs negative) } \\
\hline $\begin{array}{l}\text { Treatment modalities } \\
\text { (CCRT vs RT alone) }\end{array}$ & 0.124 & 2.939 & 0.744 & 11.604 & 0.069 & 3.000 & 0.919 & 9.793 \\
\hline $\begin{array}{l}\text { Preoperative EBRT dose } \\
\text { ( } 40-45 \text { Gy vs } 46-50 \text { Gy) }\end{array}$ & 0.177 & 0.356 & 0.080 & 1.592 & 0.094 & 0.337 & 0.095 & 1.202 \\
\hline
\end{tabular}

Abbreviations: CCRT, concurrent chemoradiotherapy; EBRT, external beam radiotherapy; HR, hazard ratio; RT, radiotherapy; OS, overall survival; PFS, progression-free survival; $\mathrm{Cl}$, confidence interval. 

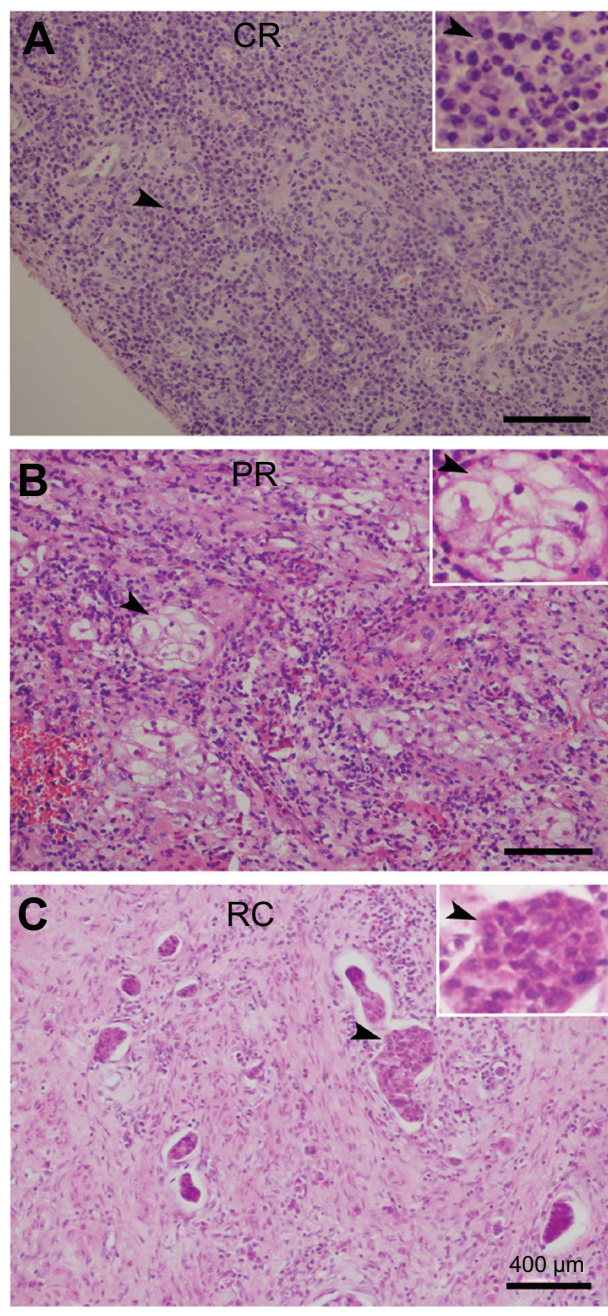

Figure 2 (A-C) Three major pathological responses, ie, complete response $(C R)$, partial response $(P R)$, and residual carcinoma $(R C)$ in patients treated with preoperative concurrent chemoradiotherapy or radiotherapy alone. CR (A) shows mainly inflammatory cell infiltration, PR (B) shows presence of persistent atypical cells or cervical intraepithelial neoplasia, and $R C(C)$ shows residual tumor tissue or tumor cells in cervical tissue.

The data of this study did not show obvious differences in CR rate between CCRT and RT alone in all 174 cases (38/121, $31.4 \%$ vs $15 / 53,28.3 \%$; $P=0.724$ ); however, CCRT showed potential benefit for CR rate enhancement (CCRT, 21/55, $38.2 \%$ vs RT alone, $8 / 26,30.8 \% ; P=0.623$ ) when tumor size was less than $5 \mathrm{~cm}$.

Treatment failures occurred in 15 patients $(8.6 \%)$ and consisted of local-regional recurrence in five patients (2.9\%) and distant metastasis in ten patients (5.7\%), mainly in the RC group (three patients with regional recurrences and seven patients with metastases). None of the regional recurrences occurred in the CR group, except for one patient with metastasis. Two patients with distant metastases and two patients with regional recurrence were observed in the PR group.
Table 3 Late complications in patients treated with preoperative CCRT or RT alone

\begin{tabular}{lll}
\hline Types of complications & $\begin{array}{l}\text { Number of } \\
\text { occurrence in } \\
\text { CCRT (\%) }\end{array}$ & $\begin{array}{l}\text { Number of } \\
\text { occurrence in } \\
\text { RT alone (\%) }\end{array}$ \\
\hline Leg edema (grade 2) & $3(2.5 \%)$ & I (I.9\%) \\
Irradiation enteritis (grade 2) & $3(2.5 \%)$ & $3(5.7 \%)$ \\
Irradiation cystitis (grade 2) & I0 (8.3\%) & $4(7.5 \%)$ \\
Uronephrosis (grade 2) & $3(2.5 \%)$ & $3(5.7 \%)$ \\
Uronephrosis (grade 3) & I (0.8\%) & 0 \\
Intestine obstruction (grade 3) & I (0.8\%) & 0 \\
Thrombus (grade 3) & $4(3.3 \%)$ & 0 \\
Renal failure (grade 3) & I (0.8\%) & 0 \\
Total & $26 / 121(2$ I.5\%) & I I/53 (20.8\%) \\
\hline
\end{tabular}

Abbreviations: CCRT, concurrent chemoradiotherapy; RT, radiotherapy.

\section{Tumor size as another prognostic factor in CCRT or RT alone}

According to Cox regression analysis, there was no statistical difference in survival between preoperative CCRT and RT alone (3-year PFS, 92.0\% vs 86.7\%, $P=0.069$; 3-year OS, $95.0 \%$ vs $88.1 \%, P=0.124$ ) for all 174 patients. Nevertheless, preoperative CCRT did have significantly improved 3-year PFS ( $P=0.032,92.0 \%$ CCRT vs $76.5 \%$ RT alone), but not OS ( $P=0.055,94.4 \%$ CCRT vs 53.6\% RT alone) compared with RT alone when tumor size was less than $5 \mathrm{~cm}$. Statistical data analysis for those 81 patients with tumor size less than $5 \mathrm{~cm}$ treated with CCRT or RT alone is shown in Figure 4.

\section{Preoperative EBRT dose analysis}

Patients with a higher preoperative EBRT dose had an improved pathological CR (40-45 Gy/20-25 f vs 46-50 Gy/23-25 f, $15 \%$ vs $35.6 \% ; P=0.002$ ), even though EBRT dose did not influence OS and PFS. Relationships between EBRT dose and local control $(P=0.704)$, lymph-node involvement $(P=0.521)$, and complications $(P=0.667)$ were all analyzed, but none of these results showed statistical significance.

\section{Discussion}

In this retrospective approach, CCRT achieved an outcome superior to RT alone in 174 Chinese patients with FIGO stage IB2-IIIB cervical carcinoma. A new finding of this study is that preoperative CCRT was associated with significantly improved PFS and OS compared with RT alone when the tumor size was less than $5 \mathrm{~cm}$. Although CCRT has become the standard of care for LACC and systemic surgery was not recommended in a routine procedure, 3-year OS (74\%) or 5-year OS or PFS $(50 \%-63 \%)$ of the standard CCRT alone were still not satisfactory. ${ }^{17}$ On the other hand, a clinical trial showed a contribution of surgery to the patients with bulky residual 


\section{A}

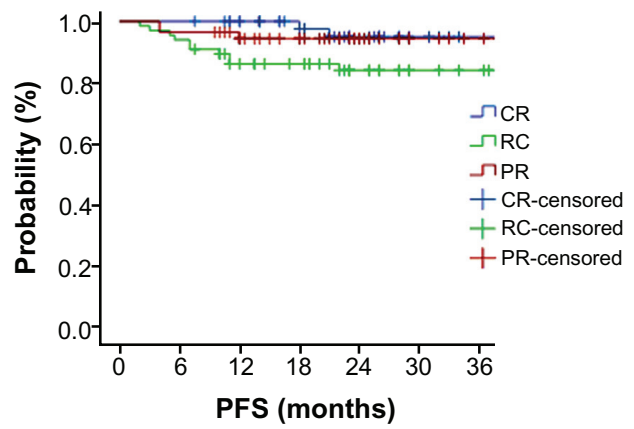

B

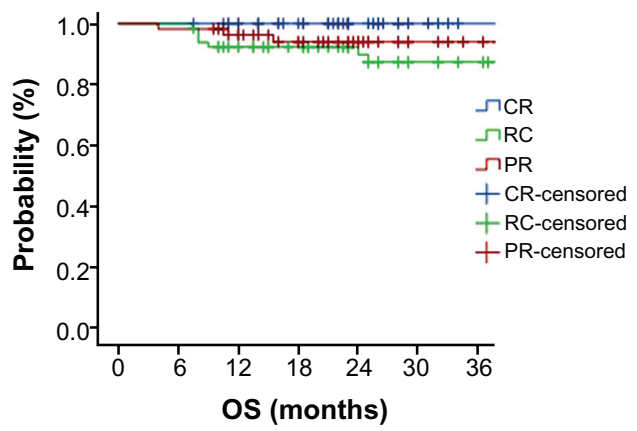

Figure 3 Survival curves of progression-free survival (PFS) (A) and overall survival (OS) (B) of local advanced cervical carcinoma patients with pathological complete response, partial response, and residual carcinoma after preoperative concurrent-chemoradiotherapy (CCRT) or radiotherapy (RT)-alone modalities.

Notes: The 3-year fellow-up indicates high PFS (94.9\%) and OS (I00\%) in the complete response (CR) group, and low PFS (84.0\%) and OS (87.3\%) in the residual carcinoma (RC) group. Statistical analysis shows significant differences of PFS and OS between the CR and RC groups $(P=0.035, C R$ vs RC for 3 -year PFS; $P=0.018, C R$ vs RC for 3-year OS).

disease after CCRT for cervical carcinoma by increasing OS and local control rate. ${ }^{18}$ We applied preoperative CCRT/RT by pelvic radiation of 40-50 Gy instead of radical radiation for the patients with LACC in this study. Interestingly, our data showed preoperative CCRT achieved better outcome in comparison to RT alone for LACC with acceptable low toxicity and complications. This study suggests that a combination of preoperative CCRT and radical surgery may provide a feasible and effective treatment for patients with LACC, though further comprehensive investigation is needed for modified concurrent chemotherapy in improved treatment of patients with late-stage or bulky tumor size.

Postoperative pathological response was highly related with OS and PFS. RC after surgery was identified as a high-risk factor for local failure. ${ }^{11,19,20}$ So far, there are no consensus criteria for postoperative pathological response classification after neoadjuvant treatment for LACC. Huguet et al found that PFS was significantly different when the size of histological residual tumor in uterine cervix was more or less than $5 \mathrm{~mm} .{ }^{21}$ Ferrandina et al observed clinical results of 161 patients with LACC treated with neoadjuvant chemoradiotherapy and radical surgery. ${ }^{22}$ PFS and OS were lower for patients with macroscopic residual disease compared to patients with histological $\mathrm{CR}$ and microscopic residual disease. Classe et al gave a similar conclusion in their analysis of 175 patients with FIGO stage IB2-IVA cervical cancer. ${ }^{20}$ In our study, patients with pathological CR had better PFS and OS compared to patients with pathological residual disease. Besides, we found that 55 patients $(31.6 \%$ of all patients) with pathological atypical cells and cervical intraepithelial neoplasia had different outcomes compared to patients with pathological CR and residual disease. The clinical significance and histological significance of atypical cells and cervical intraepithelial neoplasia after CCRT should require longer follow-up and identification of specific biomarkers. As for the dose delivered by pelvic
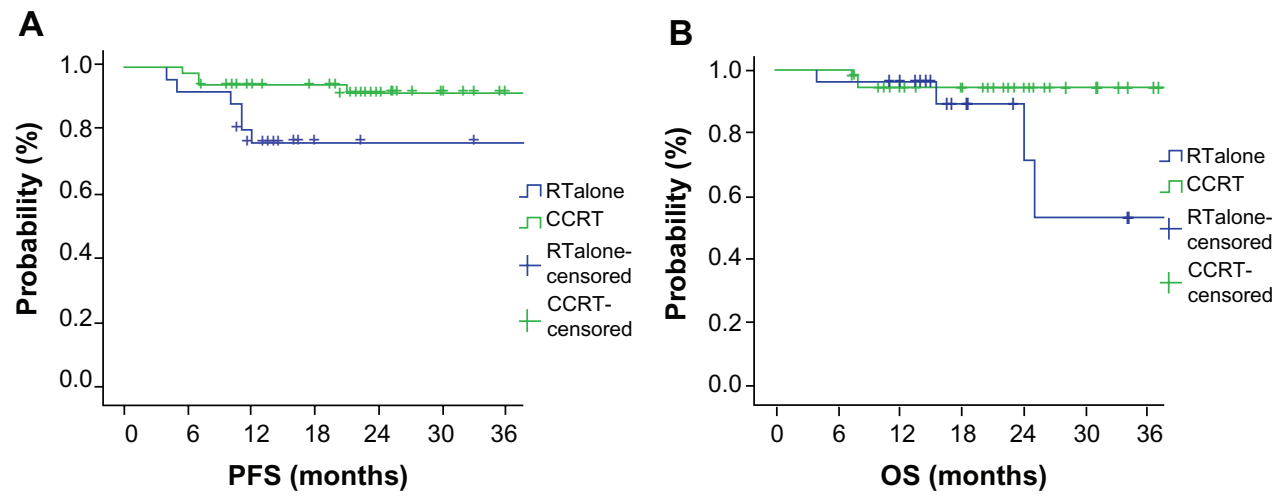

Figure 4 Survival curves of 3-year progression-free survival (PFS) (A) and overall survival (OS) (B) in 8 I local advanced cervical carcinoma patients with tumor size less than $5 \mathrm{~cm}$ and treated with preoperative concurrent-chemoradiotherapy (CCRT) or radiotherapy (RT) alone.

Notes: CCRT showed improved 3-year PFS but not OS compared with RT alone. Statistical analysis shows significant differences between CCRT and RT alone ( $P=0.032$, $92.0 \%$ CCRT vs $76.5 \%$ RT alone for 3-year PFS; $P=0.055$, CCRT vs RT alone for OS). 
EBRT, 40-45 Gy/20-25 f was reported in some previous studies, ${ }^{20,21}$ whereas we further demonstrated that a dose of 46-50 Gy/23-25 f could increase pathological CR without increasing severe complications in this study.

The precise relationship between tumor size and progression is still not clearly understood in LACC, though primary tumor diameter was regarded as one of the important prognostic factors. ${ }^{19,23-25}$ While it was shown that 5-year local control and DFS were $96 \%$ and 95\%, respectively, when tumor size was less than $5.2 \mathrm{~cm}$, while 5 -year local control and DFS were $67 \%$ and $66 \%$ when the tumor diameter was more than $5.2 \mathrm{~cm}$, Hirakawa et al reported that tumor size was an independent prognostic factor for locoregional failure and survival on multivariate analysis. ${ }^{19}$ Data from our study revealed that the OS and PFS were significantly different when the tumor size was less than $5 \mathrm{~cm}$ or $\geq 5 \mathrm{~cm}$. The results of our study are consistent with an observation by Baiocchi et al. ${ }^{26}$ They found that tumor size larger than $5 \mathrm{~cm}$ did not correlate with the risk of recurrence and death from cancer, and we further showed preoperative CCRT improved PFS and OS in comparison with that of RT alone when patients had a tumor diameter less than $5 \mathrm{~cm}$, indicating that beneficial effect of CCRT on PFS and OS of patients might be limited or depend on tumor size of cervical cancer.

In Huang et al's research, the prognostic factors contained incomplete tumor regression, a low hemoglobin level, and positive lymph-node metastasis. ${ }^{27}$ The survival state might depend on the number of lymph nodes involved: those with four or more involved lymph nodes had worse cause-specific survival compared with patients with one to three involved lymph nodes. ${ }^{28}$ Most research has focused on how to reduce the rate of pelvic lymph-node metastasis rate recently. In Huguet et al's report, pelvic lymph-node metastasis rate was reduced to $7.8 \%$ after CCRT based on cisplatin and 5-FU in IB-IIB stage cervical carcinoma. ${ }^{29}$ As a contrast, in Kirova et al and Colombo et al, after CCRT based on weekly cisplatin, the pelvic lymph-node metastasis rate was $20.0 \%$ and $22.5 \%$, respectively. ${ }^{30,31}$ For stage IB-IVA, Classee et al reported that the rate was $32.5 \%$ after CCRT based on weekly cisplatin, and this rate could be reduced to $16.1 \%$ by CCRT based on cisplatin and 5-FU in Macchia et al's study. ${ }^{32,33}$ Taken together with our observation in this study, postoperative pathologic response, tumor diameter, and lymph-node involvement might be highly correlated with clinical outcomes of patients with LACC.

In conclusion, this retrospective study has shown that CCRT followed by radical surgery achieved a better outcome compared with RT alone in LACC patients, based on pathologic response, tumor diameter, and lymph-node involvement. The novelty of this study is that preoperative CCRT was associated with significantly improved PFS and OS compared with RT alone when tumor size was less than $5 \mathrm{~cm}$. We should indicate that this observation is still retrospective and from a pilot study, which might be a limitation along with a lack of sufficiently balanced numbers of patients. Nevertheless, these findings may deserve further comprehensive investigation in a randomized clinical trial.

\section{Acknowledgments}

This work was supported in part by a grant from the National Science Foundation of China (81272346 and 30970862). Authors also thank Dr Loren K Mell, Center for Advanced Radiotherapy Technologies, Department of Radiation Oncology, University of California San Diego, CA, USA, for his critical reading and valuable suggestions on this manuscript revision.

\section{Disclosure}

The authors report no conflicts of interest in this work.

\section{References}

1. Peters WA 3rd, Liu PY, Barrett RJ 2nd, et al. Concurrent chemotherapy and pelvic radiation therapy compared with pelvic radiation therapy alone as adjuvant therapy after radical surgery in high-risk early-stage cancer of the cervix. J Clin Oncol. 2000;18:1606-1613.

2. Keys HM, Bundy BN, Stehman FB, et al. Cisplatin, radiation, and adjuvant hysterectomy compared with radiation and adjuvant hysterectomy for bulky stage IB cervical carcinoma. $N$ Engl J Med. 1999;340:1154-1161.

3. Morris M, Eifel PJ, Lu J, et al. Pelvic radiation with concurrent chemotherapy compared with pelvic and para-aortic radiation for high-risk cervical cancer. N Engl J Med. 1999;340:1137-1143.

4. Thomas GM. Improved treatment for cervical cancer: concurrent chemotherapy and radiotherapy. N Engl J Med. 1999;340:1198-1200.

5. National Cancer Institute. Clinical announcement on cervical cancer Bethesda (MD): National Institutes of Health; 1999.

6. Jemal A, Murray T, Ward E, et al. Cancer statistics, 2005. CA Cancer J Clin. 2005;55:10-30.

7. Kato S, Ohno T, Thephamongkhol K, et al. Multi-institutional phase II clinical study of concurrent chemoradiotherapy for locally advanced cervical cancer in East and Southeast Asia. Int J Radiat Oncol Biol Phys. 2010;77:751-757.

8. Póti Z, Patyánik M, Nemeskéri C. Radiochemotherapy of cervical carcinoma. (Necessity of dose reduction in chemotherapy). Orv Hetil. 2006;147:1315-1320. Hungarian.

9. Tabata T, Takeshima N, Nishida H, Hirai Y, Hasumi K. A randomized study of primary bleomycin, vincristine, mitomycin and cisplatin (BOMP) chemotherapy followed by radiotherapy versus radiotherapy alone in stage IIIB and IVA squamous cell carcinoma of the cervix. Anticancer Res. 2003;23:2885-2890.

10. Ferrandina G, Legge F, Fagotti A, et al. Preoperative concomitant chemoradiotherapy in locally advanced cervical cancer: safety, outcome, and prognostic measures. Gynecol Oncol. 2007;107:S127-S132.

11. Mariagrazia D, Anna F, Gabriella F, et al. Preoperative chemoradiotherapy in locally advanced cervical cancer: long-term outcome and complications. Gynecol Oncol. 2005;99:S166-S170. 
12. Chemoradiotherapy for Cervical Cancer Meta-Analysis Collaboration. Reducing uncertainties about the effects of chemoradiotherapy for cervical cancer: a systematic review and meta-analysis of individual patient data from 18 randomized trials. J Clin Oncol. 2008;26:5802-5812.

13. Taylor A, Rockall AG, Reznek RH, Powell ME. Mapping pelvic lymph nodes: guidelines for delineation in intensity-modulated radiotherapy. Int J Radiat Oncol Biol Phys. 2005;63:1604-1612.

14. Cox JD, Stetz J, Pajak TF. Toxicity criteria of the Radiation Therapy Oncology Group (RTOG) and the European Organization for Research and Treatment of Cancer (EORTC). Int J Radiat Oncol Biol Phys. 1995;31:1341-1346.

15. Chassagne D, Sismondi P, Horiot JC, et al. A glossary for reporting complications of treatment in gynecological cancers. Radiother Oncol. 1993;26:195-202.

16. Dindo D, Demartines N, Clavien PA. Classification of surgical complications: a new proposal with evaluation in a cohort of 6336 patients and results of a survey. Ann Surg. 2004;240:205-213.

17. Dueñas-González A, Zarbá JJ, Patel F, et al. Phase III, open-label, randomized study comparing concurrent gemcitabine plus cisplatin and radiation followed by adjuvant gemcitabine and cisplatin versus concurrent cisplatin and radiation in patients with stage IIB to IVA carcinoma of the cervix. J Clin Oncol. 2011;29:1678-1685.

18. Houvenaeghel G, Lelievre L, Buttarelli M, et al. Contribution of surgery in patients with bulky residual disease after chemoradiation for advanced cervical carcinoma. Eur J Surg Oncol. 2007;33:498-503.

19. Hirakawa M, Nagai Y, Toita T, et al. High-risk group for locoregional recurrence in patients with stage IB-IIB squamous cell carcinoma of the cervix treated with concurrent chemoradiotherapy. Anticancer Res. 2011;31:1437-1441.

20. Classe JM, Rauch P, Rodier JF, et al. Surgery after concurrent chemoradiotherapy and brachytherapy for the treatment of advanced cervical cancer: morbidity and outcome: results of a multicenter study of the GCCLCC (Groupe des Chirurgiens de Centre de Lutte Contre le Cancer). Gynecol Oncol. 2006;102:523-529.

21. Huguet F, Cojocariu OM, Levy P, et al. Preoperative concurrent radiation therapy and chemotherapy for bulky stage IB2, IIA, and IIB carcinoma of the uterine cervix with proximal parametrial invasion. Int J Radiat Oncol Biol Phys. 2008;72:1508-1515.

22. Ferrandina G, Legge F, Fagotti A, et al. Preoperative concomitant chemoradiotherapy in locally advanced cervical cancer: safety, outcome, and prognostic measures. Gynecol Oncol. 2007;107:S127-S132.
23. Lèguevaque $\mathrm{P}$, Motton $\mathrm{S}$, Delannes $\mathrm{M}$, et al. Completion surgery or not after concurrent chemoradiotherapy for locally advanced cervical cancer? Eur J Obstet Gynecol Reprod Biol. 2011;155:188-192.

24. Motton S, Houvenaeghel G, Delannes M, et al. Results of surgery after concurrent chemoradiotherapy in advanced cervical cancer: comparison of extended hysterectomy and extrafascial hysterectomy. Int J Gynecol Cancer. 2010;20:268-275.

25. Liu MT, Hsu JC, Liu WS, et al. Prognostic factors affecting the outcome of early cervical cancer treated with radical hysterectomy and post-operative adjuvant therapy. Eur J Cancer Care (Engl). 2008;17:174-181.

26. Baiocchi G, Guimaraes GC, Rosa Oliveira RA, et al. Prognostic factors in pelvic exenteration for gynecological malignancies. Eur J Surg Oncol. 2012;38:948-954.

27. Huang YT, Wang CC, Tsai CS, et al. Long-term outcome and prognostic factors for adenocarcinoma/adenosquamous carcinoma of cervix after definitive radiotherapy. Int J Radiat Oncol Biol Phys. 2011;80: 429-436.

28. Song S, Song C, Kim HJ, et al. 20 year experience of postoperative radiotherapy in IB-IIA cervical cancer patients with intermediate risk factors: Impact of treatment period and concurrent chemotherapy. Gynecol Oncol. 2012;124:63-67.

29. Huguet F, Cojocariu OM, Levy P, et al. Preoperative concurrent radiation therapy and chemotherapy for bulky stage IB2, IIA, and IIB carcinoma of the uterine cervix with proximal parametrial invasion. Int J Radiat Oncol Biol Phys. 2008;72:1508-1515.

30. Kirova YM, Bourhaleb Z, Alran S, et al. Preoperative concomitant radiochemotherapy in bulky carcinoma of the cervix: Institut Gurie experience. Cancer Radiother. 2009;13:291-297. French.

31. Colombo PE, Bertrand MM, Gutowski M, et al. Total laparoscopic radical hysterectomy for locally advanced cervical carcinoma (stages IIB, IIA and bulky stages IB) after concurrent chemoradiation therapy: surgical morbidity and oncological results. Gynecol Oncol. 2009; 14:404-409.

32. Classe JM, Rauch P, Rodier JF, et al. Surgery after concurrent chemoradiotherapy and brachytherapy for the treatment of advanced cervical cancer: morbidity and outcome: results of a multicenter study of the GCCLCC. Gynecol Oncol. 2006;102:523-529.

33. Macchia G, Ferrandina G, Deodato F, et al. Concomitant boost dose escalation plus large-field preoperative chemoradiation in locally advanced carcinoma of the uterine cervix: results of a phase I study (LARA-CC-1). Gynecol Oncol. 2010;118:128-133.
OncoTargets and Therapy

\section{Publish your work in this journal}

OncoTargets and Therapy is an international, peer-reviewed, open access journal focusing on the pathological basis of all cancers, potential targets for therapy and treatment protocols employed to improve the management of cancer patients. The journal also focuses on the impact of management programs and new therapeutic agents and protocols on

\section{Dovepress}

patient perspectives such as quality of life, adherence and satisfaction The manuscript management system is completely online and includes a very quick and fair peer-review system, which is all easy to use. Visit http://www.dovepress.com/testimonials.php to read real quotes from published authors. 